\title{
Nonequilibrium Control of Thermal and Mechanical Changes in a Levitated System
}

\author{
Markus Rademacher@, ${ }^{1,{ }^{*}+}$ Michael Konopik, ${ }^{2}$ Maxime Debiossac $\odot,{ }^{1}$ David Grass $\odot,{ }^{1, \S}$ Eric Lutz $\odot,{ }^{2}$ and Nikolai Kiesel $\odot^{1, \dagger}$ \\ ${ }^{1}$ Vienna Center for Quantum Science and Technology (VCQ), Faculty of Physics, University of Vienna, A-1090 Vienna, Austria \\ ${ }_{2}^{2}$ Institute for Theoretical Physics I, University of Stuttgart, D-70550 Stuttgart, Germany
}

(Received 4 August 2021; accepted 17 December 2021; published 15 February 2022)

\begin{abstract}
Fluctuation theorems are fundamental extensions of the second law of thermodynamics for small nonequilibrium systems. While work and heat are equally important forms of energy exchange, fluctuation relations have not been experimentally assessed for the generic situation of simultaneous mechanical and thermal changes. Thermal driving is indeed generally slow and more difficult to realize than mechanical driving. Here, we use feedback cooling techniques to implement fast and controlled temperature variations of an underdamped levitated microparticle that are 1 order of magnitude faster than the equilibration time. Combining mechanical and thermal control, we verify the validity of a fluctuation theorem that accounts for both contributions, well beyond the range of linear response theory. Our results allow the investigation of general far-from-equilibrium processes in microscopic systems that involve fast mechanical and thermal changes at the same time.
\end{abstract}

DOI: 10.1103/PhysRevLett.128.070601

Work and heat are two central quantities in thermodynamics. The energy change related to mechanical driving, that is, the variation of a system parameter such as the position of a piston, corresponds to work. On the other hand, the energy change related to thermal driving, created by a temperature difference, is associated with heat [1]. While both variables are deterministic in macroscopic systems, they become stochastic at the microscopic scale owing to the presence of thermal fluctuations [2,3]. In such systems, the second law has been generalized in the form of fluctuation theorems that account for the effects of nonnegligible fluctuations [2,3]. Fluctuation relations reveal the universal laws that govern the properties of the random nonequilibrium entropy production. Their general validity arbitrarily far from thermal equilibrium makes them particularly useful in the study of nonequilibrium systems $[2,3]$.

Fluctuation theorems for mechanical driving [4,5] have been extensively investigated experimentally in the past decades in numerous systems [6,7], ranging from biomolecules $[8,9]$ and colloidal particles $[10,11]$ to mechanical [12] and electronic [13] systems. By contrast, only relatively few experimental studies have been devoted to fluctuation relations for thermal driving [14], based, for instance, on a varying bath temperature [15] or a fixed temperature difference between two systems $[16,17]$. This

Published by the American Physical Society under the terms of the Creative Commons Attribution 4.0 International license. Further distribution of this work must maintain attribution to the author(s) and the published article's title, journal citation, and DOI. reflects the fact that thermal control is generally slow and more difficult to implement than mechanical control [18]. The effective bath temperature has, for example, been modulated using laser absorption [15,19], mechanical [16], and electrical [20-22] random forcing. However, no fluctuation theorem for simultaneous mechanical and thermal drivings has been studied experimentally yet, despite its relevance in many areas where such changes occur at the same time [23-28], including the important case of microscopic heat engines [19-21].

In this Letter, we experimentally demonstrate fast thermal and mechanical control of an oscillator on timescales much shorter than its relaxation time. We use our system to investigate for the first time generalized fluctuation relations that account for simultaneous mechanical and thermal changes far from equilibrium [23-28]. To this end, we use optically trapped microparticles [29-31]. Here, the harmonic trap created by the optical potential can be dynamically controlled and the effective environmental temperature of the particle's center-of-mass motion can be set by linear feedback cooling [32] (Fig. 1). While recent studies have examined the effect of information gain on the thermodynamics of a particle interacting with a constant temperature bath in the presence of feedback [33-38], we consider in the following the dynamics of a particle coupled to a bath with an effective time-dependent temperature. Both situations are physically distinct and the corresponding fluctuation relations are fundamentally different. Specifically, we test two protocols (Fig. 1): first, solely the temperature is modified (protocol $P_{1}$ ) and, second, both temperature and spring constant are varied (protocol $P_{2}$ ). With our approach, we are able to study nonequilibrium processes that occur 1 order of magnitude faster than the 


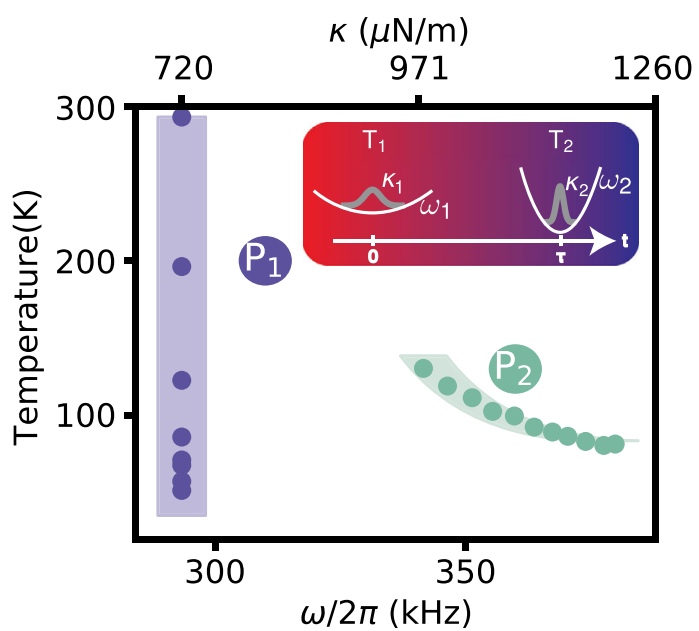

FIG. 1. Nonequilibrium processes with fast thermal and mechanical changes. We realize changes of the center-of-mass temperature $T$ and of the spring constant $\kappa$ (corresponding to frequency $\omega$ ) of a harmonically trapped levitated microparticle by varying the feedback gain and the laser trap power (inset). Protocol $P_{1}$ (purple) corresponds to a thermal change with fixed spring constant, while protocol $P_{2}$ (turquoise) refers to simultaneous thermal and mechanical changes. Full circles are data, with error bars smaller than the symbol size. Shaded areas represent the temperatures set experimentally, taking into account uncertainty due to laser power drifts. In the inset, the gray lines show position distributions during protocol $P_{2}$ when the temperature is decreased from $T_{1}$ to $T_{2}$ and the frequency is simultaneously increased from $\omega_{1}$ to $\omega_{2}$ during a time $\tau$.

relaxation time of the system $\left(\gamma_{\mathrm{p}}^{-1}\right)$. In this regime, linear response theory is no longer applicable. Our findings emphasize the importance of the thermal (also known as entropic) work, associated with a change of entropy of the bath [39], on the same footing as the conventional mechanical work [40]. Fluctuation theorems for thermal driving indeed only permit accurate determination of the equilibrium free energy difference when this novel contribution is included.

The generalized fluctuation relation for mechanical and thermal drivings of a system described by the Hamiltonian $H(\kappa)$, with time-dependent parameter $\kappa(t)$, and inverse temperature $\beta(t)$ reads [23-28]

$$
\langle\exp (-W)\rangle=\exp [-\Delta(\beta F)]
$$

where $\Delta(\beta F)=\beta(\tau) F(\tau)-\beta(0) F(0)$ with $F$ the (equilibrium) free energy of the system. The generalized dimensionless work $W$ is defined as [24-28]

$W=W_{\text {mech }}+W_{\text {ther }}=\int_{0}^{\tau} d t \beta \partial H / \partial \kappa \dot{\kappa}+\int_{0}^{\tau} d t \dot{\beta} H$.

The first term in Eq. (2) corresponds to the dimensionless stochastic mechanical work along a single trajectory of duration $\tau$, while the second term is the random thermal (or entropic) work induced by a temperature change [40]. The brackets $\langle$.$\rangle denote an average over many trajectories.$ The dimensionless heat exchanged with the bath follows from a generalized first law as $\int \beta \delta Q=\Delta(\beta H)-W$ with $\Delta(\beta H)=\beta(\tau) H(\tau)-\beta(0) H(0)$ [23]. Equation (1) reduces to the usual Jarzynski equality in the case of constant temperature, $\dot{\beta}=0$.

For a harmonic Hamiltonian, $H(x, p, \kappa)=p^{2} / 2 m+$ $\kappa x^{2} / 2$, with position $x$, momentum $p$, mass $m$, and spring constant $\kappa$, like in our experiment, the normalized free energy difference may be evaluated explicitly. We find

$$
\Delta(\beta F)_{\mathrm{ho}}=\ln [\beta(\tau) / \beta(0)]+\ln [\kappa(\tau) / \kappa(0)] / 2 .
$$

The fluctuation theorem [Eq. (1)] holds arbitrarily far from equilibrium. Close to equilibrium, in the linear response regime, Eq. (1) may be Taylor expanded [2,3]. To first order, one recovers the equilibrium result, $\Delta(\beta F)_{\mathrm{eq}}=\langle W\rangle$, whereas, to second order, one obtains the linear-response formula, $\Delta(\beta F)_{\mathrm{lr}}=\langle W\rangle-\left(\left\langle W^{2}\right\rangle-\langle W\rangle^{2}\right) / 2$, where $\left\langle W^{2}\right\rangle-$ $\langle W\rangle^{2}$ is the variance of the total work.

The harmonic oscillator in our experiment is a levitated silica microsphere with a diameter of $969 \mathrm{~nm}$. It is optically trapped by two counterpropagating laser beams at $1064 \mathrm{~nm}$ in the intensity maximum of a standing wave [Fig. 2(a)] inside a hollow-core photonic crystal fiber (HCPCF) [43]. The amplitude of the microsphere's center-of-mass motion is much smaller than the wavelength of the trapping laser; thus, the optical potential is approximately harmonic with a tunable power-dependent frequency $\omega_{p} / 2 \pi$ between $250 \mathrm{kHz}$ and $400 \mathrm{kHz}$. The gas surrounding the microsphere acts as a heat bath at room temperature. Its coupling to the microparticle's center-of-mass motion is determined by the pressure, which we set to 1.5 mbar. This results in a coupling rate of $\gamma_{p} / 2 \pi=6.3 \mathrm{kHz}$, which is much smaller than the mechanical frequency. Hence, the underdamped Langevin equation for an harmonic oscillator applies [40].

To perform work on the system and thus implement mechanical driving, we control the spring constant $\kappa$ via the optical trap power $P$, exploiting their linear dependence $(\kappa \propto P)$. We further realize thermal control of the microparticle center-of-mass temperature via feedback control, i.e., cold damping [32]. To implement feedback cooling, we exert a radiation pressure force from an additional laser that counteracts the particle motion. Specifically, we apply a delayed, linearly position-dependent force $F_{\mathrm{fb}}=$ $-g m \gamma_{p} \omega_{0} x\left(t-t_{\mathrm{fb}}\right)$, where $g$ is the feedback gain, $\omega_{0}=$ $\sqrt{\kappa / m}$ the mechanical frequency without feedback, $m$ the mass of the microsphere, and $t_{\mathrm{fb}}=5 \pi / 2 \omega_{0}$ the feedback delay [40]. The feedback force may be split into a position and a velocity component [40]. The velocity component provides additional friction and therefore cools the temperature of the particle motion. The effective inverse temperature is then given by $\beta(t)=\beta_{0}[1+g \sin (\Delta \phi)]$, where 


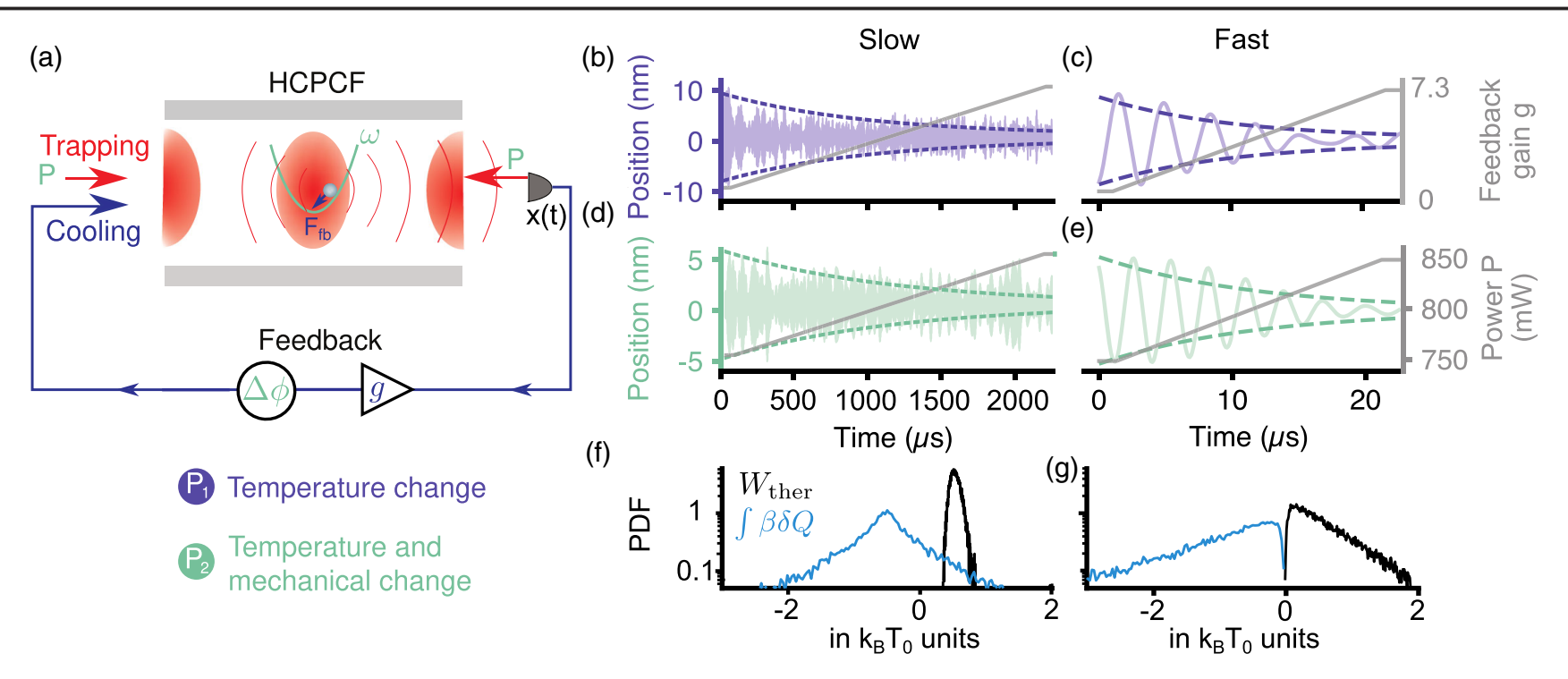

FIG. 2. Experimental setup and single trajectories. (a) Schematic of the experimental setup. Two counterpropagating laser beams (red arrows) of wavelength $1064 \mathrm{~nm}$ trap a $969 \mathrm{~nm}$ silica particle at the intensity maximum of the standing wave formed inside a hollow-core photonic crystal fiber (HCPCF). The scattered light (red lines) along the HCPCF provides the center-of-mass motion $x(t)$ of the particle. An additional feedback laser beam (blue arrow) cools the center-of-mass motion of the particle. Here, the velocity contribution of the feedback force $F_{\mathrm{fb}}=g \gamma_{p} m \sin (\Delta \phi) \dot{x}$ depends on the feedback gain $g$ and the trapping laser power $P$ via the feedback phase $\Delta \phi$ (see the Supplemental Material [40]). Protocol $P_{1}$ (thermal change, purple) is implemented by a linear increase of the feedback gain $g$ at constant optical trap power. Protocol $P_{2}$ (thermal and mechanical change, turquoise) is realized by changing the optical trap power $P$. (b)-(e) Measured single-particle trajectories (solid lines) and ensemble variances (dashed lines, based on 15000 trajectories) of the particle center-of-mass motion are plotted together with the corresponding change of the respective control parameter (gray lines). Panels (b), (d) and (c),(e) correspond to the slowest equilibrium (fastest nonequilibrium) protocols, 1 order of magnitude slower (faster) than the relaxation time of the system. (f)- $(\mathrm{g})$ Experimental distributions of the dimensionless thermal work $W_{\text {ther }}$ (black) and dimensionless heat $\int \beta \delta Q$ (blue) in the case of protocol $P_{2}$, for slow (f) as well as fast (g) drivings.

$\Delta \phi=\omega t_{\mathrm{fb}}$ is the phase introduced by the delayed feedback, with $\omega$ the mechanical frequency with feedback [40]. We carry out protocol $P_{1}$ (thermal driving) by increasing the gain $g(t)$ linearly in time to solely vary the temperature, while the optical potential remains unchanged. We furthermore implement protocol $P_{2}$ (mechanical and thermal drivings) by tuning the laser power of the trap, hence the mechanical frequency $\omega(t)$, and keeping the gain $g$ constant. This also changes the effective inverse bath temperature $\beta(t)$ due to the frequency dependence of the phase $\Delta \phi$. In both cases, we measure the center-of-mass motion along the axis of the hollow-core fiber by interferometric readout of the light scattered by the microparticle [40,43].

Figures 2(b)-(e) display recorded single-particle trajectories for protocols $P_{1}$ (purple) and $P_{2}$ (turquoise) for slow (equilibrium) and fast (nonequilibrium) drivings: the solid (dashed) lines indicate the particle center-of-mass motion (ensemble variance). During protocol $P_{1}$, the feedback gain $g$ is varied linearly from 0 to 7.3 (gray lines), decreasing the particle temperature by a factor of up to $\approx 8.3$ (Fig. 1). During protocol $P_{2}$, the laser trapping power $P$ is increased linearly from 750 to $850 \mathrm{~mW}$, reducing the spring constant by $\approx 11 \%$, and decreasing the temperature by a factor $\approx 1.5$ (Fig. 1). Parameters are chosen to ensure stability and repeatability of the experiment over the whole measurement time [40]. Note that light absorption does not significantly influence the particle temperature in our case [40]. Between each cycle and before the protocol ramp starts, the system equilibrates to the inverse effective temperature $\beta(0)$, determined by the feedback. The duration $\tau$ of slow (fast) protocols is $2.26 \mathrm{~ms}(22.6 \mu \mathrm{s})$. With $\gamma_{p}^{-1} \approx 0.2 \mathrm{~ms}$, equilibrium protocols thus probe the particle dynamics in the quasistatic regime where $\tau \gg \gamma_{p}^{-1}$. By contrast, nonequilibrium protocols are an order of magnitude faster than the relaxation time $\tau \ll \gamma_{p}^{-1}$ of the system.

We evaluate the random mechanical and thermal works, $W_{\text {mech }}$ and $W_{\text {ther }}$, together with the dimensionless stochastic heat, $\int \beta \delta Q$, along 15000 single trajectories. Figures 2(f), (g) show the distributions of the dimensionless thermal work $W_{\text {ther }}$ (black) and dimensionless heat $\int \beta \delta Q$ (blue) for protocol $P_{2}$ (the corresponding distribution for $W_{\text {mech }}$ is presented in the Supplemental Material [40]). For slow driving (f), the dimensionless thermal work has a Gaussian profile whereas the dimensionless heat distribution exhibits exponential tails, in analogy to the case of pure mechanical driving [44]. On the other hand, for fast driving (g), the two distributions are clearly asymmetric. However, their exact shape is yet unknown. 

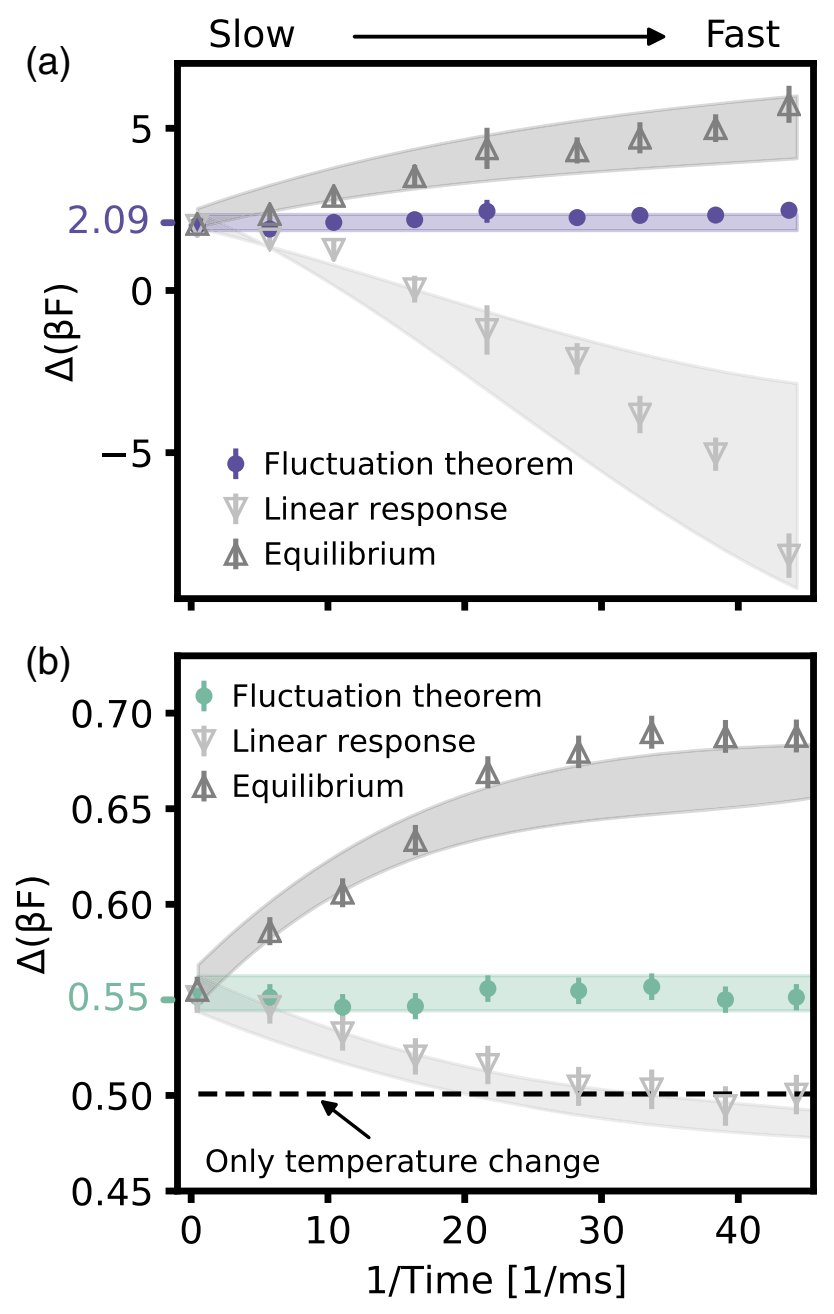

FIG. 3. Fluctuation theorem for thermal and mechanical modulations. Normalized free energy difference $\Delta(\beta F)$ versus inverse driving time $\tau$ for (a) thermal protocol $P_{1}$ and (b) thermal and mechanical protocol $P_{2}$. Dots represent experimental data evaluated using Eq. (1). Shaded areas are theoretical predictions with an uncertainty that incorporates long term laser drifts [40]. Errors bars are determined by the standard deviation over 15000 runs. Equilibrium, $\Delta(\beta F)_{\mathrm{eq}}$, (triangle up) and linear response, $\Delta(\beta F)_{\mathrm{lr}}$, (triangle down) results only hold for slow driving. The horizontal dashed line in (b) indicates the contribution of thermal change only.

We further investigate, for both protocols, the normalized free energy difference $\Delta(\beta F)$ as a function of the inverse driving time associated with the speed of the protocol. We compare three methods to determine $\Delta(\beta F)$ from our experimental data: by applying the fluctuation theorem $\Delta(\beta F)_{\mathrm{ft}}$ [Eq. (1)] (dots), by using the equilibrium result $\Delta(\beta F)_{\mathrm{eq}}$ (triangle up), and by employing the linear response formula $\Delta(\beta F)_{\mathrm{lr}}$ (triangle down). In addition, we show the calculated free energy difference $\Delta(\beta F)_{\text {ho }}$ as given by Eq. (2).

For the purely thermal control implemented in protocol $P_{1}$ [Fig. 3(a)], we theoretically expect $\Delta(\beta F)_{\mathrm{ho}}=$ $2.09 \pm 0.24$. This value is inferred from equilibrium

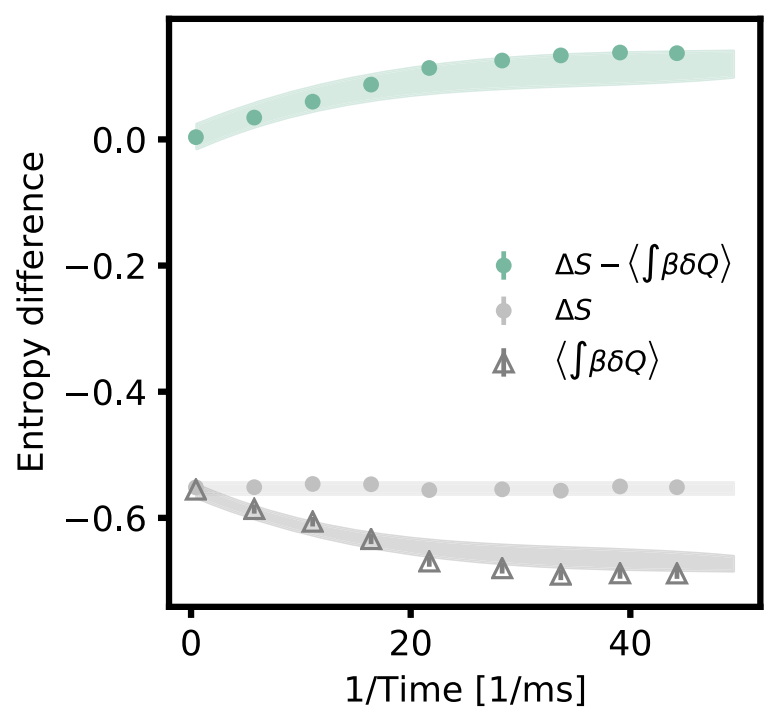

FIG. 4. Clausius inequality for thermal and mechanical changes. Comparison between the measured (dimensionless) heat exchanged with the environment $\left\langle\int \beta \delta Q\right\rangle$ (triangles) and the (dimensionless) entropy variation of the system $\Delta S$ (gray full circles), as a function of the inverse protocol time for thermal and mechanical changes (protocol $P_{2}$ ) followed by an isothermal thermalization. The Clausius inequality, $\Delta S-\left\langle\int \beta \delta Q\right\rangle \geq 0$, is verified for any protocol speed. Shaded areas are theoretical predictions that include the uncertainty on the underlying experimental parameters. Error bars are determined via the standard deviation of the respective value over 15000 runs and are smaller than the symbol size.

measurements of the system temperature and spring constant using Eq. (3). Uncertainties on the theoretical prediction originate from slow drifts of these parameters and are shown as purple areas in the plot [40]. We observe that the equilibrium and linear response results, $\Delta(\beta F)_{\mathrm{eq}}$ and $\Delta(\beta F)_{\text {lr }}$, hold for long protocol times. However, both rapidly deviate from $\Delta(\beta F)_{\text {ho }}$ for faster protocols. By contrast, we have very good agreement with the values obtained from the fluctuation relation [Eq. (1)] [purple dots in Fig. 3(a)], within the error bars, for all driving speeds. Even for the fastest protocol, we determine a value consistent with our expectation $\Delta(\beta F)_{\mathrm{ft}}=2.47 \pm 0.25$. Figure 3(b) shows similar results for the combined mechanical and thermal control implemented in protocol $P_{2}$ : while the equilibrium and near-equilibrium approximations are valid for slow changes, the nonequilibrium fluctuation relation [Eq. (1)] (turquoise dots) correctly reproduces the equilibrium free energy difference $\Delta(\beta F)_{\mathrm{ho}}=$ $0.55 \pm 0.01$ (turquoise area), even very far from the quasistatic regime with $\Delta(\beta F)_{\mathrm{ft}}=0.55 \pm 0.01$ for the fastest protocol. The dashed black line represents the theoretical normalized free energy difference when only thermal work is taken into account. As it significantly departs from the actual value in the experiment, we conclude that our protocol actually requires accounting for both mechanical work and entropic work. 
The fluctuation relation [Eq. (1)] extends the Clausius statement of the second law to stochastic far-fromequilibrium processes. Noting that $\Delta(\beta F)=\Delta(\beta H)_{\mathrm{eq}}-$ $\Delta S$, where $\Delta S$ is the (dimensionless) entropy change between initial and final equilibrium states, and using the convexity of the exponential, we indeed have $\left\langle\int \beta \delta Q\right\rangle \leq \Delta S$ [23]. The integrated heat absorbed by the system, divided by the temperature at which that heat is absorbed, is thus bounded from above by the entropy variation of the system. Figure 4 presents the first experimental confirmation of that fundamental inequality in microscopic systems for protocol $P_{2}$ followed by an isothermal equilibration, for varying protocol speed. The inequality is verified for arbitrarily far-from-equilibrium processes. Contrary to the generalized fluctuation theorem [Eq. (1)], which holds exactly as an equality, the Clausius inequality provides a worse bound for increasing protocol speeds.

In conclusion, we have demonstrated an experimental route to implement fast and controlled temperature changes in an underdamped levitated harmonic system using feedback cooling techniques. We have exploited the ability to realize simultaneous mechanical and thermal drivings on timescales much shorter than the equilibration time of the system, to reveal the importance of the entropic work and extend the applicability of fluctuation theorems, for both mechanical and entropic works, beyond the linear response regime. Our versatile experimental approach enables the study of generic nonequilibrium transformations in microscopic systems that involve fast mechanical and thermal modulations at the same time. The implementation of fast temperature control is not limited to our system and applies generally to oscillators controlled by linear feedback. Given the recent success in cooling mechanical oscillators into their ground state of motion with linear feedback cooling [45-47], the extension of such fluctuation relations to the quantum domain appears to be an exciting prospect [40].

We thank Markus Aspelmeyer for his support and valuable discussions. N.K. acknowledges support from the Austrian Science Fund (FWF): Y 952-N36, START. D. G. acknowledges support through the doctoral school Complex Quantum Systems (CoQuS, W1210). We further acknowledge financial support from the German Science Foundation (DFG) under Project FOR 2724. We also acknowledge the support of NVIDIA Corporation with the donation of the Titan Xp GPU used for this research.

* Corresponding author.

m.rademacher.18@ucl.ac.uk

Corresponding author.

nikolai.kiesel@univie.ac.at

${ }^{\ddagger}$ Present address: Department of Physics and Astronomy, University College London, London WC1E 6BT, United Kingdom.
${ }^{\S}$ Present address: Department of Chemistry, Duke University, Durham, North Carolina 27708, USA.

[1] A. B. Pippard, Elements of Classical Thermodynamics (Cambridge University Press, Cambridge, 1964).

[2] C. Jarzynski, Equalities and inequalities: Irreversibility and the second law of thermodynamics at the nanoscale, Annu. Rev. Condens. Matter Phys. 2, 329 (2011).

[3] U. Seifert, Stochastic thermodynamics, fluctuation theorems and molecular machines, Rep. Prog. Phys. 75, 126001 (2012).

[4] C. Jarzynski, Nonequilibrium Equality for Free Energy Differences, Phys. Rev. Lett. 78, 2690 (1997).

[5] G. E. Crooks, Entropy production fluctuation theorem and the nonequilibrium work relation for free energy differences, Phys. Rev. E 60, 2721 (1999).

[6] S. Ciliberto, R. Gomez-Solano, and A. Petrosyan, Fluctuations, linear response, and currents in out-of-equilibrium systems, Annu. Rev. Condens. Matter Phys. 4, 235 (2013).

[7] S. Ciliberto, Experiments in Stochastic Thermodynamics: Short History and Perspectives, Phys. Rev. X 7, 021051 (2017).

[8] J. Liphardt, S. Dumont, S. B. Smith, I. Tinoco, and C. Bustamante, Equilibrium information from nonequilibrium measurements in an experimental test of Jarzynski's equality, Science 296, 1832 (2002).

[9] D. Collin, F. Ritort, C. Jarzynski, S. B. Smith, I. Tinoco, and C. Bustamante, Verification of the Crooks fluctuation theorem and recovery of RNA folding free energies, Nature (London) 437, 231 (2005).

[10] G. M. Wang, E. M. Sevick, E. Mittag, D. J. Searles, and D. J. Evans, Experimental Demonstration of Violations of the Second Law of Thermodynamics for Small Systems and Short Time Scales, Phys. Rev. Lett. 89, 050601 (2002).

[11] V. Blickle, T. Speck, L. Helden, U. Seifert, and C. Bechinger, Thermodynamics of a Colloidal Particle in a Time-Dependent Nonharmonic Potential, Phys. Rev. Lett. 96, 070603 (2006).

[12] F. Douarche, S. Joubaud, N. B. Garnier, A. Petrosyan, and S. Ciliberto, Work Fluctuation Theorems for Harmonic Oscillators, Phys. Rev. Lett. 97, 140603 (2006).

[13] O.-P. Saira, Y. Yoon, T. Tanttu, M. Möttönen, D. V. Averin, and J.P. Pekola, Test of the Jarzynski and Crooks Fluctuation Relations in an Electronic System, Phys. Rev. Lett. 109, 180601 (2012).

[14] C. Jarzynski and D. K. Wójcik, Classical and Quantum Fluctuation Theorems for Heat Exchange, Phys. Rev. Lett. 92, 230602 (2004).

[15] J. R. Gomez-Solano, A. Petrosyan, and S. Ciliberto, Heat Fluctuations in a Nonequilibrium Bath, Phys. Rev. Lett. 106, 200602 (2011).

[16] A. Bérut, A. Imparato, A. Petrosyan, and S. Ciliberto, Stationary and Transient Fluctuation Theorems for Effective Heat Fluxes between Hydrodynamically Coupled Particles in Optical Traps, Phys. Rev. Lett. 116, 068301 (2016).

[17] S. Ciliberto, A. Imparato, A. Naert, and M. Tanase, Heat Flux and Entropy Produced by Thermal Fluctuations, Phys. Rev. Lett. 110, 180601 (2013).

[18] Y. Dubi and M. Di Ventra, Colloquium: Heat flow and thermoelectricity in atomic and molecular junctions, Rev. Mod. Phys. 83, 131 (2011). 
[19] V. Blickle and C. Bechinger, Realization of a micrometresized stochastic heat engine, Nat. Phys. 8, 143 (2012).

[20] I. A. Martínez, É. Roldán, L. Dinis, D. Petrov, J. M. Parrondo, and R. A. Rica, Brownian Carnot engine, Nat. Phys. 12, 67 (2016).

[21] J. Roßnagel, S. T. Dawkins, K. N. Tolazzi, O. Abah, E. Lutz, F. Schmidt-Kaler, and K. Singer, A single-atom heat engine, Science 352, 325 (2016).

[22] I. A. Martínez, E. Roldán, L. Dinis, D. Petrov, and R. A. Rica, Adiabatic Processes Realized with a Trapped Brownian Particle, Phys. Rev. Lett. 114, 120601 (2015).

[23] C. Jarzynski, Microscopic analysis of Clausius-Duhem processes, J. Stat. Phys. 96, 415 (1999).

[24] R. Chelli, S. Marsili, A. Barducci, and P. Procacci, Generalization of the Jarzynski and Crooks nonequilibrium work theorems in molecular dynamics simulations, Phys. Rev. E 75, 050101(R) (2007).

[25] R. Chelli, S. Marsili, A. Barducci, and P. Procacci, Numerical verification of the generalized Crooks nonequilibrium work theorem for non-Hamiltonian molecular dynamics simulations, J. Chem. Phys. 127, 034110 (2007).

[26] C. Chatelain, A temperature-extended Jarzynski relation: Application to the numerical calculation of surface tension, J. Stat. Mech. (2007) P04011.

[27] S. R. Williams, D. J. Searles, and D. J. Evans, Nonequilibrium Free-Energy Relations for Thermal Changes, Phys. Rev. Lett. 100, 250601 (2008).

[28] R. Chelli, Nonequilibrium work relations for systems subject to mechanical and thermal changes, J. Chem. Phys. 130, 054102 (2009).

[29] J. Gieseler and J. Millen, Levitated nanoparticles for microscopic thermodynamics: A review, Entropy 20, 326 (2018).

[30] J. Gieseler, R. Quidant, C. Dellago, and L. Novotny, Dynamic relaxation of a levitated nanoparticle from a nonequilibrium steady state, Nat. Nanotechnol. 9, 358 (2014).

[31] T. M. Hoang, R. Pan, J. Ahn, J. Bang, H. T. Quan, and T. Li, Experimental Test of the Differential Fluctuation Theorem and a Generalized Jarzynski Equality for Arbitrary Initial States, Phys. Rev. Lett. 120, 080602 (2018).

[32] T. Li, S. Kheifets, D. Medellin, and M. G. Raizen, Measurement of the instantaneous velocity of a Brownian particle, Science 328, 1673 (2010).

[33] J. M. R. Parrondo, J. M. Horowitz, and T. Sagawa, Thermodynamics of information, Nat. Phys. 11, 131 (2015).

[34] S. Toyabe, T. Sagawa, M. Ueda, E. Muneyuki, and M. Sano, Experimental demonstration of information-to-energy conversion and validation of the generalized jarzynski equality, Nat. Phys. 6, 988 (2010).
[35] J. V. Koski, V. F. Maisi, T. Sagawa, and J. P. Pekola, Experimental Observation of the Role of Mutual Information in the Nonequilibrium Dynamics of a Maxwell Demon, Phys. Rev. Lett. 113, 030601 (2014).

[36] E. Roldan, I. A. Martinez, J. M. R. Parrondo, and D. Petrov, Universal features in the energetics of symmetry breaking, Nat. Phys. 10, 457 (2014).

[37] M. Ribezzi-Crivellari and F. Ritort, Large work extraction and the Landauer limit in a continuous Maxwell demon, Nat. Phys. 15, 660 (2019).

[38] M. Debiossac, D. Grass, J. J. Alonso, E. Lutz, and N. Kiesel, Thermodynamics of continuous non-Markovian feedback control, Nat. Commun. 11, 1360 (2020).

[39] G. E. Crooks, Excursions in Statistical Dynamics (University of California, Berkeley, 1999).

[40] See Supplemental Material at http://link.aps.org/ supplemental/10.1103/PhysRevLett.128.070601 for a description of the experimental setup as well as the evaluation of the thermodynamic quantities, which includes Refs. [41,42].

[41] U. Delić, M. Reisenbauer, K. Dare, D. Grass, V. Vuletić, N. Kiesel, and M. Aspelmeyer, Cooling of a levitated nanoparticle to the motional quantum ground state, Science 367 , 892 (2020).

[42] J. Millen, T. Deesuwan, P. Barker, and J. Anders, Nanoscale temperature measurements using non-equilibrium brownian dynamics of a levitated nanosphere, Nat. Nanotechnol. 9, 425 (2014).

[43] D. Grass, J. Fesel, S. G. Hofer, N. Kiesel, and M. Aspelmeyer, Optical trapping and control of nanoparticles inside evacuated hollow core photonic crystal fibers, Appl. Phys. Lett. 108, 221103 (2016).

[44] S. Joubaud, N. Garnier, and S. Ciliberto, Fluctuation theorems for harmonic oscillators, J. Stat. Mech. (2007) P09018.

[45] M. Rossi, D. Mason, J. Chen, Y. Tsaturyan, and A. Schliesser, Measurement-based quantum control of mechanical motion, Nature (London) 563, 53 (2018).

[46] L. Magrini, P. Rosenzweig, C. Bach, A. Deutschmann-Olek, S. G. Hofer, S. Hong, N. Kiesel, A. Kugi, and M. Aspelmeyer, Real-time optimal quantum control of mechanical motion at room temperature, Nature (London) 595, 373 (2021).

[47] F. Tebbenjohanns, M. L. Mattana, M. Rossi, M. Frimmer, and L. Novotny, Quantum control of a nanoparticle optically levitated in cryogenic free space, Nature (London) 595, 378 (2021). 